\title{
Wikipedia rival calls in the experts
}

The current incarnation of Wikipedia is both phenomenally successful and, in the eyes of some critics, fundamentally flawed. The online encyclopaedia now includes more than a million entries in English alone. Although anyone can edit any article, its accuracy, at least on science topics, is surprisingly high. But Wikipedia has never given experts special standing when it comes to determining content. And that, critics say, deters the people who ought to be contributing from doing so.

Just how big a drawback that is will now be tested, with the launch of an online encyclopaedia that will give privileged status to scientists and other experts. Citizendium, a pilot version of which is due to go live in the next week, will use all of Wikipedia's content but will host it at another website (http://citizendium.org) and edit it differently. Editors with appropriate academic qualifications will have the power to settle disputes about wording, for example, and stamp articles they perceive to be accurate as 'approved'.

"One reason we are setting this up is to give scientists and other scholars a new organizational framework to clean up and improve on the work started by Wikipedia," says Larry Sanger, a philosopher and co-founder of Wikipedia, who is the driving force behind Citizendium. "Wikipedia is now the first stop for many people in their search for information on scientific topics. Many scientists would like to help make sure this resource remains accurate, but they have no desire to navigate the treacherous waters of Wikipedia's editorial system, which accords them no official role."

Reactions from the many bloggers who track the progress

"Many scientists of Wikipedia have to navigate the struggle to define treacherous waters what constitutes of Wikipedia's expertise, and of Wikipedia's that arguments editorial system." about content will be replaced by arguments over who is or isn't an expert. Others think Citizendium will find it hard to attract regular contributors, as the prospect of having an edit overruled by a higher power will not appeal.

But scientists who contribute regularly to Wikipedia say Citizendium has promise. "I like the idea notionally," says Vaughan Bell of the Institute of Psychiatry in London, who contributes to Wikipedia's schizophrenia page, among others. William Connolley of the British Antarctic Survey in Cambridge, UK, who updates Wikipedia climate entries, adds that some scientists have become frustrated with Wikipedia because of the difficulty in agreeing edits, although both he and Bell agree that conflict can sometimes result in better articles.

Jim Giles 